\title{
Motion Estimation of Elastic Articulated Objects from Image Points and Contours
}

\author{
Hailang Pan, Yuncai Liu, and Lei Shi \\ Institute of Image Processing and Pattern Recognition, \\ Shanghai Jiao Tong University, P.R. China 200030 \\ \{panhailang, whomliu, sl0030322014\} asjtu.edu.cn
}

\begin{abstract}
This paper presents two new methods of elastic articulated objects modeling and motion estimation based on a new revolving conic surface. The deformation of human body surfaces can be represented by adjusting only one or two deformation parameters for each limb. Then, the 3D deformation and motion parameters are estimated by corresponding $2 \mathrm{D}$ image points and contours from a sequences of stereo images.
\end{abstract}

\section{Introduction}

Elastic articulated objects are the combination of articulated objects and nonrigid objects. Human body is a typical elastic articulated objects. In the past researches, a number of method for human deformable body modeling have been proposed. Plankers[1]developed a framework for 3D shape and motion recovery of articulated deformable objects. Smooth implicit surfaces, known as metaballs, are attached to an articulated skeleton of the human body and are arranged in an anatomically-based approximation. Sminchisescu [2] built a human body model which consists of kinematic 'skeletons', covered by 'flesh' built from superquadric ellipsoids. A typical model has 9 deformable shape parameters for each body part. Apuzzo[3] presents simplified Model of a Limb. Only three ellipsoidal metaballs are attached to each limb skeleton. Each limb has twelve deformation parameters.

Our research focuses on two major points: 3D human body modeling and its parameters determination from images. The proposed human limb model is composed of two layers: a skeleton layer and a body surface layer.The body surface layer is expressed by revolving conic surfaces. We can deform the body surface layer during animation by adjusting only one or two deformation parameters for each human limb. 3D human arm parameters determination includes the skeleton motion parameters estimation and the deformable surfaces deformation parameters determination.

\section{Establishment of Revolving Conic Surfaces and Human Skeleton}

A 3D conic curve can be obtained by intersecting a conic surface with a plane that does not go through the vertex of the conic surface or by intersecting two conic surfaces. Consequently, a 3D conic curve S can be specified by the system of equations

$$
\left\{\begin{array}{l}
f_{1}\left(x_{S}, y_{S}, z_{S}\right)=0 \\
f_{2}\left(x_{S}, y_{S}, z_{S}\right)=0
\end{array}\right.
$$


Where the equation $\mathrm{f}_{1}\left(\mathrm{x}_{\mathrm{S}}, \mathrm{y}_{\mathrm{S}}, \mathrm{z}_{\mathrm{S}}\right)$ is a $3 \mathrm{D}$ conic surface and the equation $\mathrm{f}_{2}\left(\mathrm{x}_{\mathrm{S}}, \mathrm{y}_{\mathrm{S}}, \mathrm{z}_{\mathrm{S}}\right)$ is a $3 \mathrm{D}$ plane or a $3 \mathrm{D}$ conic surface.

See Fig. 1 , let $\mathrm{P}_{0}=\left(\mathrm{x}_{0}, \mathrm{y}_{0}, \mathrm{z}_{0}\right)$ be a point on the $3 \mathrm{D}$ straight line $\mathrm{L}$ which can represents the skeleton of a limb. Let e $(m, n, p)$ be a non-zero vector parallel to the straight line $\mathrm{L}$, then the straight line $\mathrm{L}$ has the canonical equation of the form

$$
\frac{x-x_{0}}{m}=\frac{y-y_{0}}{n}=\frac{z-z_{0}}{p}=t
$$

Let the curve $\mathrm{S}$ be a $3 \mathrm{D}$ conic curve. We can obtain a revolving conic surface by revolving the $3 \mathrm{D}$ conic curve $\mathrm{S}$ around the $3 \mathrm{D}$ straight line $\mathrm{L}$. A revolving conic surface can represent the human body surface of a limb. It is defined as follows: Let $\mathrm{P}(\mathrm{x}, \mathrm{y}, \mathrm{z})$ be a arbitrary point on the revolving conic surface. Let the plane $\mathrm{U}$, passing through the point $\mathrm{P}$ and perpendicular to the straight line $\mathrm{L}$. The point $\mathrm{P}_{\mathrm{S}}\left(\mathrm{x}_{\mathrm{S}}, \mathrm{y}_{\mathrm{S}}, \mathrm{z}_{\mathrm{S}}\right)$ is the intersection of the plane $U$ and the conic curve $S$. The point $O\left(x_{L}, y_{L}, z_{L}\right)$ is the intersection of the plane $U$ and the straight line $L$. Then the plane $U$, passing through the point $\mathrm{P}, \mathrm{P}_{\mathrm{S}}, \mathrm{O}$ and perpendicular to the straight line $\mathrm{L}$, has the equation of the form

$$
m\left(x-x_{S}\right)+n\left(y-y_{S}\right)+p\left(z-z_{S}\right)=0
$$

The points $\mathrm{P}, \mathrm{P}_{\mathrm{S}}, \mathrm{O}$ satisfy the relation $\overline{P O}=\overline{P_{S} O}$, namely

$$
\left(x-x_{L}\right)^{2}+\left(y-y_{L}\right)^{2}+\left(z-y_{L}\right)^{2}=\left(x_{S}-x_{L}\right)^{2}+\left(y_{S}-y_{L}\right)^{2}+\left(z_{S}-z_{L}\right)^{2}
$$

Since the point $\mathrm{P}_{\mathrm{S}}\left(\mathrm{x}_{\mathrm{S}}, \mathrm{y}_{\mathrm{S}}, \mathrm{z}_{\mathrm{S}}\right)$ lies on the conic curve $\mathrm{S}$, its coordinates satisfy the equation (1). Hence, the system of equations (1),(3),(4) are the revolving conic surface.

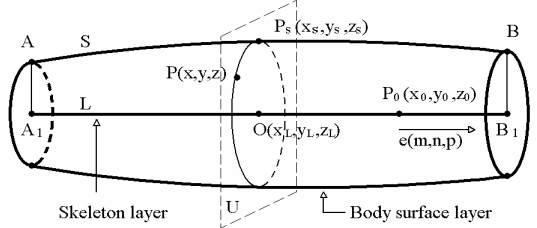

Fig. 1. 3D Revolving conic surface

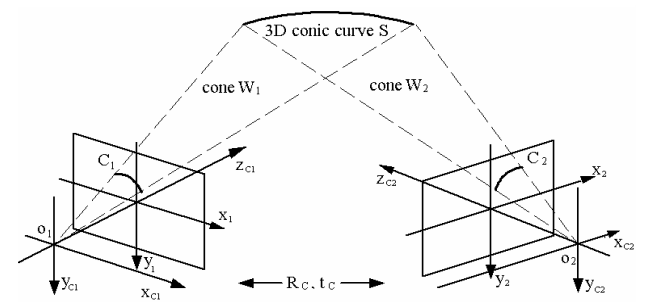

Fig. 2. Stereo coordinate system of a $3 \mathrm{D}$ conic curve

The human skeleton system is treated as a series of jointed links(segments), which can be modeled as a articulated body. We describe the body as a stick model consisting of a set of fifteen joints (plus the head) connected by fourteen body segments [4].

\section{Human Arm Modeling and Its Parameters Determination}

\subsection{Modeling from Image Points and Its Deformation Parameters Determination}

A 3D conic curve $S$ can be specified by the system of equations

$\left\{\begin{array}{l}\mathrm{f}_{1}\left(x_{S}, y_{S}, z_{S}\right)=\left(\mathrm{a}_{0}+\mathrm{a}_{1} \mathrm{x}_{S}+\mathrm{a}_{2} \mathrm{y}_{S}+\mathrm{a}_{3} \mathrm{z}_{S}\right)\left(\mathrm{b}_{0}+\mathrm{b}_{1} \mathrm{x}_{S}+\mathrm{b}_{2} \mathrm{y}_{S}+\mathrm{b}_{3} \mathrm{z}_{S}\right)-\rho\left(\mathrm{u}_{0}+\mathrm{u}_{1} \mathrm{x}_{S}+\mathrm{u}_{2} \mathrm{y}_{S}+\mathrm{u}_{3} \mathrm{z}_{S}\right)^{2}=0 \\ \mathrm{f}_{2}\left(x_{S}, y_{S}, z_{S}\right)=\mathrm{f}_{\mathrm{ABC}}\left(x_{S}, y_{S}, z_{S}\right)=a \cdot x_{S}+b \cdot y_{S}+c \cdot z_{S}+d=0\end{array}\right.$ 
The first equation is a 3D conic surface. The second equation is a plane passing through the vertices A, B, C. Hence, the system of equations (3),(4),(5) are a revolving conic surface, which can represent deformable human body surface.

The input of our system of equations is $(\mathrm{x}, \mathrm{y}, \mathrm{z}), 3 \mathrm{D}$ coordinate of one arbitrary feature point on the revolving conic surface. The expected output is the parameter $\rho$ that describes the model's deformation. Hence the deformation of one limb can also be determined with three feature points, two of them are points A, B and the third is a arbitrary point on the deformable surface. This is also a nonlinear system of equations, which can be solved by the Levenberg-Marquardt nonlinear least squares.

\subsection{Modeling from Contours and Its Deformation Parameters Determination}

The geometry of the stereo is shown in Fig. 2. Suppose there is a $3 \mathrm{D}$ conic curve $\mathrm{S}$ in space. Its two projections on two images are represented by two quadratic form $\mathrm{C}_{1}$ and $\mathrm{C}_{2}[5]$ :

$$
C_{i}\left(x_{i}, y_{i}\right)=X_{i}^{T} Q_{i} X_{i}=0 \quad i=1,2
$$

where $X_{i}=\left[x_{i}, y_{i}, 1\right]^{T} .\left(x_{i}, y_{i}\right)$ are the $2 D$ coordinates in two images.

Let $\mathrm{X}_{\mathrm{w}}=\left[\mathrm{x}_{\mathrm{w}}, \mathrm{y}_{\mathrm{w}}, \mathrm{Z}_{\mathrm{w}}, 1\right]^{\mathrm{T}} .\left(\mathrm{x}_{\mathrm{w}}, \mathrm{y}_{\mathrm{w}}, \mathrm{Z}_{\mathrm{w}}\right)$ is a point in the world coordinate system. Then $\mathrm{X}_{\mathrm{i}}$ and $X_{w}$ are related to each other through the following equation:

$$
z_{c i} X_{i}=M_{i} X_{w}
$$

Substituting the equation (7) into the equation (6), we obtain the representations of the two cones $\mathrm{W}_{1}$ and $\mathrm{W}_{2}$ passing through the $2 \mathrm{D}$ conic curve $\mathrm{C}_{1}$ (or $\mathrm{C}_{2}$ ) and the camera center $\mathrm{o}_{1}\left(\right.$ or $\left.\mathrm{O}_{2}\right)$ :

$$
\left\{\begin{array}{l}
W_{1}\left(x_{w}, y_{w}, z_{w}\right)=X_{w}^{T} M_{1}^{T} Q_{1} M_{1} X_{w}=0 \\
W_{2}\left(x_{w}, y_{w}, z_{w}\right)=X_{w}^{T} M_{2}^{T} Q_{2} M_{2} X_{w}=0
\end{array}\right.
$$

The $3 \mathrm{D}$ conic curve $\mathrm{S}$ in space is the intersection curve of two cones $\mathrm{W}_{1}$ and $\mathrm{W}_{2}$. Hence, the system of equations (3),(4),(8) are a revolving conic surface, which can represent deformable human body surface.

We fit one 2D conic curve to the contour of each limb in left or right images. We directly minimize the implicit form of a $2 \mathrm{D}$ conic curve to determine the best values of the deformation paramete $\rho$.We can get two deformation parameters $\rho_{\text {i.left }}$ and $\rho_{i \text { iright }}$ of each limb at a time.

\subsection{Motion Parameters Estimation of Human Skeleton}

We pick up the centers of the circles that pass through the vertices of the conic curve and are tangent to the boundary of each limb. We regard the points, lying on the sticks that linking these centers, as the skeleton points. The motion of one limb can be estimated with only three skeleton points correspondences[6].

\section{Experiment Results}

The controlled scenes are acquired by using a stereoscopic vision system. In the experiment of modeling from image points, six paper markers have been sticked on the 
body surfaces of the arm, three on the upper arm and three on the lower arm. On each limb, we detect three paper markers. The 3D coordinates of these feature points, acquired from the image planes, are used as the input data for determining the deformation parameters of two limbs. The estimated results are listed in Table 1 that describe the model's deformation. $\rho_{1}$ and $\rho_{2}$ are the estimated deformation parameters of the two limbs respectively. In the experiment of modeling from image contours, the human arm is nicely distinguishable against the simpler background using edge detection. The estimated results are listed in Table 2 that describe the model's deformation. Fig. 3 depicts the movement of human arm. We use OpenGL code to visualize the recovered 3D model.

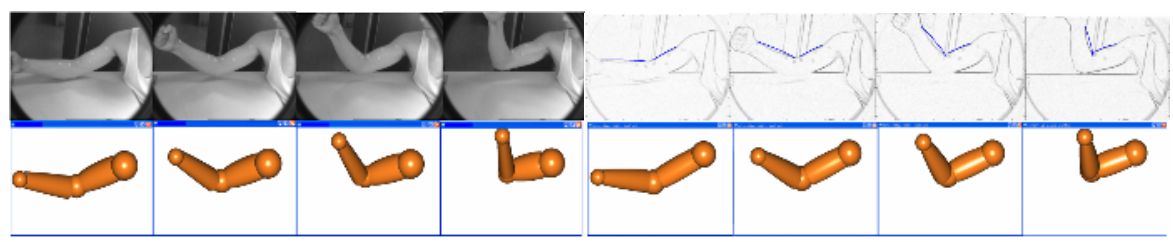

Fig. 3. Left first row: original images of left camera. Left second row: estimated 3D models from image points. Right first row: corresponding target contours and conic curve fitting results. Right second row: estimated 3D model from image contours.

Table 1. Estimated deformation parameters of modeling from image points

\begin{tabular}{|c|l|l|l|l|}
\hline deformation parameter & Frame1 & Frame2 & Frame3 & Frame4 \\
\hline$\rho_{1}$ & 0.5639 & 0.7995 & 1.5288 & 2.3358 \\
\hline$\rho_{2}$ & 0.0727 & 0.1775 & 0.0736 & 0.0052 \\
\hline
\end{tabular}

\section{Conclusion}

We have presented two new methods of elastic articulated objects (human bodies) modeling based on a new revolving conic surface. Our experiments have demonstrated that our model can express the deformation of human body surfaces properly.

\section{References}

1. Ralf Plankers and Pascal Fua. Articulated Soft Objects for Multiview Shape and Motion Capture. IEEE Transaction on PAMI, 2003, 25 (9): $1182 \sim 1187$.

2. ESTIMATION ALGORITHMS FOR AMBIGUOUS VISUAL MODELS (C.Sminchisescu), Doctoral Thesis, INRIA, July 2002.

3. D'Apuzzo N, Plänkers R, Gruen A, Fua F and Thalmann D, Modelling Human Bodies from Video Sequences, Proc. Electronic Imaging1999, San Jose, California, January. 
4. Xiaoyun Zhang, Yuncai Liu and TS Huang. "Articulated Joint Estimation from Motion Using Two Monocular Images", Pattern Recognition Letters 25(10): 1097-1106, 2004.

5. Songde MA.Conics-Based Stereo, Motion Estimation, and Pose Determination. Intern. J. Computer Vision, Vol. 10, No.1, 1993.

6. Xiaoyun Zhang, Yuncai Liu and TS Huang. " Motion Estimation of Articulated Objects from Perspective Views", LNCS, Vol. 2492, pp.165-176 ,2002 . 